\title{
HACIA UN MODELO SISTÉMICO DE EVALUACIÓN DEL SISTEMA EDUCATIVO
}

"Mira con los ojos de todo el mundo y no habrá nada que no puedas ver. Escucha con las orejas de todo el mundo y no habrá nada que no puedas escuchar. Piensa con la mente de todo el país y no habrá nada que no puedas conocer" (Sun Bin, El arte de la guerra II)

\section{Albert Arbós Bertran \\ Vicedecano de Magisterio de la Universidad Internacional de Catalunya, España}

En este breve escrito queremos posicionarnos en favor de un modelo sistémico de evaluación del sistema educativo, en un contexto globalizador -de la información ${ }^{1}$, de la economía-, pero que, a su vez, tienen necesidad de no perder las raíces nacionales que le distinguen de sus vecinos, en un contexto en que la necesidad de autonomía y de planificación se complementen de forma positiva para dar respuesta a las demandas de la sociedad de nuestro tiempo. Creemos que este modelo es el que mejor puede ayudar a avanzar la formación en alternancia, facilitándole un camino hacia la calidad ${ }^{2}$. Analizaremos, brevemente, el posicionamiento actual de los países de América Latina.

La eficacia, la equidad y la calidad que se reclaman a los servicios públicos por parte de los usuarios han llevado a las administraciones educativas -incluyendo las latinoamericanas- de la mayor parte del planeta a políticas de regulación y control (accountability) a través de la evaluación en una de sus funciones -ni la única ni la más importante-, la de controlar. Pero, las mismas administraciones, a través de las leyes, han dado un papel, cada vez más creciente, a la autonomía de las instituciones escolares y de las organizaciones educativas, indispensable, en parte, porqué la complejidad de la educación hoy en día impide su control como en tiempos pretéritos, ámbito en el que Latinoamérica debe avanzar decididamente. Hay una clara tendencia al equilibrio entre el control de la Administración central y la descentralización hacia los organismos educativos periféricos y, en última instancia, los establecimientos educativos. Países más centralistas, en tiempos pretéritos, se orientan hacia formas variadas de descentralización (España, Francia, Suecia, Argentina -1992-, Paraguay -2º Plan de Desarrollo Educacional 1983-1994-, Venezuela, Colombia), y, al contrario, países descentralizados, centralizan el control (Inglaterra, México -lal SEP creó el Sistema Nacional de Evaluación Educacional-, El Salvador -des de 1989, a través de la Oficina de Planificación-, Bolivia, que descentralizó en el período 1980-1990, ha vuelto a la centralización en el período 1990-1997) y, por último, otra tendencia es la de aquellos países (Bélgica, Holanda, Finlandia, Chile -en el ámbito de los proyectos de municipalización del currículum-, Nicaragua -Estrategia Nacional de Educación de 1999-) que se orientan hacia la autonomía de las instituciones educativas ${ }^{3}$, aunque buena parte de ellos pretenden, al menos desde el punto de vista teórico, conciliar la centralización con la descentralización (Brasil Secretarías Estaduales y Municipales por un lado y el MEC/INEP, por otro, Cuba, Guatemala -Ley de

\footnotetext{
${ }^{1}$ Queda explicitado en la Declaración de Santo Domingo, de julio de este año, donde los ministros y ministras de educación, abogan por la incorporación de las nuevas tecnologías de la información y la comunicación a la educación para "no permanecer al margen de las vertiginosas transformaciones que el mundo contemporáneo está viviendo".

2 El concepto de "calidad", sin más concreciones, es un mito con una fuerte implantación en las sociedades postindustriales. Apostamos por un concepto de calidad dinámico y tranformador, que busca la excelencia de cada persona y de cada institución en sí misma. Y, en lo colectivo, unida al cocncepto de equidad, para lograr la construcción de una sociedad más justa y más humana.

${ }^{3}$ OCDE (1995): Gros pla sur les écoles . Paris: OCDE
} 
Educación Nacional de 1991-, Uruguay -Control del sistema a través del CODICEN y descentralización como objetivo de futuro).

Las afirmaciones precedentes deberían sustentarse en un nuevo paradigma evaluativo emergente $^{4}$, que se refleja en los siguientes principios:

a)Primacía de las finalidades, es decir, la necesidad de conseguir los objetivos propuestos. Este principio nos lleva a pasar de una lógica centrada en los inputs -recursos- a una lógica centrada en los resultados y en los procesos que los producen.

b)Principio de imputabilidad, es decir, pedir responsabilidades. Se exige que las instituciones educativas y el sistema educativo en conjunto, dé cuentas a la sociedad de su eficacia y efectividad. Por eso, debe procurarse, a todos los niveles del sistema educativo, que la evaluación interna vaya asociada a la evaluación externa.

c)Principio de subsidiariedad. Ha de procurarse que las decisiones se tomen en el mismo nivel en que deban aplicarse o, al menos, que participen en las mismas. Este es un principio fundamental en el modelo sistémico.

d)Principios de autoorganización y autodesarrollo, es decir, de autonomía de funcionamiento de las instituciones y organizaciones. Cada institución educativa, cada organización educadora, debe ser un núcleo fundamental de reflexión y de innovación educativas. La reflexión a través de la evaluación debe estar al servicio de la calidad educativa.

Sobre estas bases debería construirse un modelo integrador de dos realidades: el sistema educativo y las instituciones educativas. El elemento aglutinador de estas dos realidades lo realiza la evaluación, que debe cambiar de rol; debe pasar del control a la mejora de la calidad -objetivo asimilado y explicitado, en el ámbito teórico, por la práctica totalidad de los países latinoamericanos-, de actuar en algunos niveles del sistema educativo a actuar en todos, y cambiar de función: dejar de ser acreditadora y pasar a ser generadora de los principios educativos que evalua. Este tipo de evaluación necesita un soporte técnico $\mathrm{y}$, en consecuencia, generar nuevos procedimientos evaluativos y nuevos instrumentos de desarrollo y de evaluación que se han de construir desde un punto de vista técnico, pero también desde un punto de vista social y cultural. Un nuevo modelo que nos permita establecer nuevas estrategias de evaluación que no sólo tenga por objeto la regulación y el desarrollo del sistema -tradicionalmente encargado a la Inspección o Supervisión- sino que, por encima de todo, ha de permitir la rendición de cuentas -accountability- por parte de las instituciones educativas y/o los niveles de la administración responsables de la puesta en marcha de las políticas diseñadas (no podemos olvidar que evaluar es una forma de hacer política y, por eso, hay que explicar las políticas por medio de las cuales hemos conseguido objetivos o no los hemos conseguido).

Este modelo también ha de verificar que los contenidos y los objetivos de la educación responden a las necesidades, intereses y capacidades de bs alumnos. Para conseguirlo es necesario reforzar las unidades responsables de la evaluación o bien crear otras nuevas, no tan sólo en el ámbito de la

\footnotetext{
${ }^{4}$ Mateo, J. (2000): "L’avaluació de les institucions educatives". I Jornadas sobre Medición y Evaluación Educativas: Estándares e Indicadores para analizar la realidad educativa. Valencia, marzo.
} 
Administración central, sino también a escala regional o local. La supervisión en este contexto verá reforzadas y ampliadas sus competencias.

El reto que debe plantearse América latina, de hecho ya lo ha explicitado -una vez más, ya que desde los años 50 ya venían explicitando diversos países- en la Declaración de Santo Domingo de julio de 2002, en estos momentos, y que se planteó Europa en la década de los noventa, es la de mejorar la calidad de la educación que ofrecen las instituciones educativas en su conjunto, por un lado, y, por otro, utilizar la evaluación para potenciar los sistemas educativos como respuesta a los cambios mundiales ${ }^{5}$. Y, todo ello, en un marco de globalización, internacionalización lo llama Schriewer ${ }^{6}$, en que las relaciones de interacción y de intercambio son globales, donde hay interconexión mundial en los campos de la comunicación y donde existe una harmonización transnacional de modelos y estructuras sociales. De hecho, estas relaciones ya se están dando en el campo de la Cooperación internacional en evaluación ${ }^{7}$, al frente de los cuales se sitúan Brasil, Colombia, Chile y Venezuela.

La evaluación es el resultado de las interacciones del gobierno, la sociedad educativa no gubernamental, la comunidad de especialistas de la evaluación y el mercado, cada uno con su cuota de poder. Las interacciones de estos sectores de la sociedad moderna -el Estado, la sociedad civil y la economía de mercado ${ }^{8}$ - son muy complejas, interdependientes y, frecuentemente, de carácter conflictivo, porqué, en el fondo, lo que se reparten son cuotas de poder.

\section{EVALUACIÓN DEL SISTEMA Y POLÍTICA EDUCATIVA}

La evaluación del sistema educativo debe ser un elemento de información de las autoridades públicas. Los tres niveles del sistema educativo -central, regional y local- están dominados, en la planificación, por las políticas educativas; en la administración, por la gestión científica y en la enseñanza, por el psicologismo. Partiendo de esta base, y teniendo en cuenta la naturaleza política de toda evaluación como la que planteamos, es necesario que la escuela -destinatario último y principal de la mejora del sistema educativo- sea el eje de esta evaluación, teniendo en cuenta las estructuras burocráticas y la planificación educativo-política. Estamos convencidos del papel que juegan las estructuras gubernamentales sobre el rendimiento de las escuelas, hasta el punto que la burocracia, en muchos casos, es la causa principal de su fracaso.

Se trata de analizar la eficacia de las grandes políticas que se ponen en marcha en el ámbito de la educación. Debemos buscar, de forma prioritaria, la relación entre la existencia (o amplitud) de una política y los resultados en el plano de la educación ${ }^{9}$, o, como dice Kallen ${ }^{10}$, de forma explícita "toda política,

\footnotetext{
${ }^{5}$ Gipps, C. (1998): "La evaluación del alumno y el aprendizaje en una sociedad en evolución". Perspectivas, vol.XXVIII, $n^{\circ}$.1. París: UNESCO

${ }^{6}$ Schriewer, J. (1996): "Sistema mundial y redes de interrelación: la internacionalización de la educación y el papel de la investigación comparada". Globalización y descentralización de los sistemas educativos. Barcelona: Pomares-Corredor

${ }^{7}$ Tiana, A. (2000): Avaliaçâo Educacional: Cooperación internacional en evaluación de la educación en América Latina y el Caribe: análisis de la situación y propuestas de actuación. Cúpula das Américas - Linha 2. INEP. Ministerio Da Educaçâo.

${ }^{8}$ Driessen, G. (1999): "La mejora de la igualdad y la calidad en la enseñanza: el caso de Holanda”. Revista de Educación, nº319. Madrid: MEC

${ }_{9}^{9}$ Herpen, M. van (1992): "Les indicateurs de l'enseignement: modèles théoriques". L'OCDE et les indicateurs internationaux de l'enseignement: una cadre d'analyse. Paris: OCDE
} 
toda reforma, ha de comportar su propia evaluación para que tenga pleno impacto en la política y en la práctica educativa" (Kallen,1996:7).

Dos elementos conceptuales enmarcan esta necesidad: la ya mencionada de l'accountability, es decir, que intenta garantizar una supervisión más estrecha de la tarea de las escuelas y, en el fondo, garantizar la calidad educativa de los resultados - productos-, por parte del Estado; y, la que considera más apropiada para América Latina, en estos momentos, de la responsiveness, entendida como la capacidad de dar respuesta a las necesidades del entorno -objetivo al que contribuyen de manera decisiva y decidida las CEFFAs-, y que se desarrolla en cuatro lineas fundamentales:

? Descentralización, como redistribución de poder entre el centro y la periferia

? Participación social en el gobierno de los sistemas educativos; protagonismo que debe atribuirse a la comunidad educativa (profesores, familias, estudiantes, organizaciones educativas, etc.)

? Apertura a los agentes económicos, $\mathrm{y}$,

? Autonomía de los centros educativos.

Estas cuatro líneas pretenden acercar los usuarios, en el sentido más amplio de la palabra, a la toma de decisiones relativas a los procesos y a los productos educativos.

Antes de plantear el modelo, un último concepto: la noción de transparencia. Noción enteramente política según la cual los gobiernos, las sociedades o colectividades tienen, desde un punto de vista democrático, el derecho de observar el funcionamiento de los sistemas ${ }^{11}$. $\mathrm{Y}$, como complemento a la explicación anterior, la idea que un sistema educativo moderno, dentro de una sociedad plural, no puede funcionar eficazmente si no se toman en consideración las voces y las necesidades de los clientes del mismo, de sus opiniones, sus formas de organizarse, en definitiva, el protagonismo de la sociedad civil.

\section{PROPUESTA DE UN MODELO INTEGRADOR (ANEXO 1)}

Retomando el marco teórico diseñado hasta el momento, hay que hacer algunas precisiones del marco socioeducativo para entender en su profundidad el modelo presentado. En los últimos años, tanto en Europa como en América han sido frecuentes los programas y las instituciones dedicadas a control y la mejora de lo que denominamos calidad educativa. Lo mismo en sistemas centralizados, que en sistemas descentralizados, las autoridades educativas han puesto en marcha mecanismos de evaluación para recoger información, que con frecuencia ha recaído, única y exclusivamente, sobre los resultados escolares -el SAEB en Brasil, Colombia y Chile, en el marco del proyecto internacional TIMSS, o Venezuela en el RLS-PIRLS, entre otros-, por tanto, en los alumnos y, en menor medida, sobre el resto del sistema educativo, con la finalidad de saber lo que pasa en el sistema educativo y dar explicaciones de los éxitos y fracasos en este ámbito educativo.

\footnotetext{
${ }^{10}$ Kallen, D. (1996): "Nouveaux paradigmes éducatifs et nouvelles politiques d'évaluation”. Évaluer et réformer les systèmes éducatifs Paris: OCDE

${ }^{11}$ OCDE (1994,1998): Education at a Glance. Paris: OCDE
} 
La globalización de la economía, primero, y la de la información después, nos han llevado a la globalización de la educación y al fortalecimiento de las instituciones internacionales en este ámbito, como IEA $^{12}$, OCDE $^{13}$, OEI, IAEP ${ }^{14}$, OREALC-UNESCO $^{15}$, que están invirtiendo muchos recursos para responder a los retos que se les presentan. Debemos colaborar con este primer nivel internacional en todo tipo de investigaciones que puedan ayudarnos a mejorar, a comprender aquellos objetivos que como nación ya tenemos planteados.

La globalización que comporta el nivel anterior no puede hacernos perder de vista la contextualización -enraízamiento nacional- de todo sistema educativo, de toda organización educativa, de todo centro educativo, de todo sujeto receptor y emisor de la educación. ¿Podemos producir persones desarraigadas de su entorno? No!. Será necesario, pues, construir un marco educativo desde nuestro contexto sociocultural más inmediato para acercarnos a la universalización de la información -objetivo que consiguen de forma acertada las CEFFAs-. La información nos viene de todas partes, pero el conocimiento se forma en nuestro interior, en el interior de cada persona en contacto con su entorno. Por eso, las políticas educativas han de estar enraizadas en las preocupaciones sociales y la eficacia en los resultados. Este es el objetivo de los niveles nacional, regional y local y su, ineludible, interrelación.

En el ámbito nacional, los órganos deberían coordinarse con un Organismo Superior de Educación, con la finalidad de compartir experiencias en el ámbito del diagnóstico y de la investigación, con el resto de los niveles educativos, y poder asesorar a los órganos legislativos correspondientes.

En el ámbito regional y/o departamental, el control sobre las escuelas debería ejercerlo el inspector o supervisor, que elevaría sus informes al parlamento regional. En este rivel, proponemos un Consejo Asesor con amplia representación de todos los implicados en la educación, y con la ayuda de expertos de la universidad, de los centros, de expertos internacionales,...

En el ámbito del centro educativo, conviene integrar la evaluación interna y la externa, ayudados por el supervisor. El centro debería dar cuentas al Consejo Escolar y a la comunidad local, a través del Consejo Escolar Municipal.

Hay un elemento clave para el correcto y eficaz funcionamiento del modelo que presentamos: la gestión de la información; los flujos de información que deben permitirnos tomar las decisiones correctas, a todos los niveles. Son muy útiles en una sociedad informatizada que permite gestionar los datos con gran rapidez. Ahí detectamos un claro déficit -brecha lo llaman algunos autores ${ }^{16}$, en el desarrollo de las nuevas tecnologías en las últimas décadas, en América Latina.

En primer lugar conviene señalar que la información debe recaer sobre todos los elementos del sistema y ha de permitir tomar decisiones sobre la práctica educativa en el ámbito de escuela y sobre la legislación en el ámbito de los órganos políticos. Esta recogida de datos debe hacerse a través de indicadores, consensuados al máximo por todos los implicados en el proceso. Es fundamental que esta información sea útil para las investigaciones que se estén llevando a cabo a todos los niveles del sistema educativo y que se produzca un feed-back en las dos direcciones -de arriba abajo y de abajo a arriba-.

\footnotetext{
12 IEA: International Association for the Evaluation of Educational Achievement

${ }^{13}$ OCDE: Organización para el Desarrollo y la Cooperación Europeas

14 IAEP: International Assessment of Educational Progress

${ }^{15}$ OREALC: Oficina Regional de Educación para América Latina y el Caribe

${ }^{16}$ Ferranti y Perry (2002): "La brecha educativa en América Latina". La Vanguardia, 14 de noviembre del 2002
} 


\section{¿UNA UTOPÍA? ¿DÓNDE NOS ENCONTRAMOS EN ESTE MOMENTO?}

Se ha recorrido un largo camino, pero el camino que queda, es apasionante. Algunos afirman que más que una época de cambios estamos en un cambio de época; si es así, conviene recordar que una época nueva plantea retos nuevos y requiere nuevas soluciones. Es indudable que América latina se encuentra, además, inmersa, en diversos grados, en una crisis económica fuerte; la tentació podría ser la de "desinvertir" en educación para priorizar otros ámbitos sociales. Lejos de esta tentación, creo que debería invertirse, decididamente, en educación y tecnología, ambas formando un todo indisoluble.

La apuesta sencilla, y económicamente barata, es la de dar un salto de calidad en los sistemas educativos y pasar de la descentralización a la autonomía de los centros educativos, nudo gordiano de la evaluación sistémica del sistema educativo que presentamos. Entre el Estado y el centro educativo, cabe reconocer el valor de la sociedad educativa civil que, a través de organizaciones privadas sin afán de lucro, ofrecen a los ciudadanos formas educativas contextualizadas, flexibles, innovadoras y de calidad.

Analicemos, pues, el estado actual en América Latina, de algunos de los puntos más importantes de nuestro modelo.

a) Como hemos señalado al inicio de este escrito, muchos países de América Latina, ya se planteaban la mejora de la calidad educativa en los años 50 , cuando algunos países europeos no la habían hecho (España). Pero ha sido en los años 90 cuando han adquirido carta de naturaleza los sistemas de evaluación de la calidad de la educación: Brasil y Chile a finales de los 80 , Honduras (1990), Colombia (1991), República Dominicana (1992), Argentina y El Salvador (1993), México (1994) Costa Rica, Paraguay y Venezuela (1995), Bolivia (1996) ${ }^{17}$, Panamá (1996), Ecuador (Sistema Integral de Evaluación Educativa), Nicaragua (Estrategia Nacional de Educación, 1999). El primer paso en un largo recorrido consiste en querer caminar, y éste ya lo han dado buena parte de los países.

b) Un segundo aspecto que plantea el modelo es la cooperación internacional. En éste ámbito, hay mucho camino por recorrer. Excepto Colombia, Brasil, Chile y Venezuela, que participan en cuatro o más proyectos internacionales, el resto de países, debe aprovecharse mejor de las posibilidades que ofrece esta cooperación internacional, en el ámbito de costos compartidos, de personal técnico capacitado y de desarrollo de los sistemas educativos nacionales. En un segundo vagón podemos encontrar Argentina, México, Paraguay y Perú, que participan en tres proyectos internacionales. El resto, participan, e incluso en alguna ocasión sin llegar al final del proyecto, en uno o dos proyectos. En último término, la cooperación internacional debe ayudar a los responsables de la formulación de las políticas educativas a situar, a través de la comparación de los sistemas educativos, su sistema nacional de educación para implementar las reformas necesarias en las instituciones educacionales para satisfacer las necesidades de los ciudadanos de hoy y de mañana. También las organizaciones educativas privadas deben participar, a través del

\footnotetext{
${ }^{17}$ Wolff, L. (1998): Las Evaluaciones Educacionales en América Latina: Avance Actual y Futuros Desafíos. Whashington \& Santiago de Chile: PREAL
} 
Estado correspondiente, o, en nombre propio, en estudios y proyectos internacionales para mejorar, mediante la comparación y la innovación, ya que deben ser, de forma permanente, organizaciones que aprenden.

c) Un tercer aspecto es el papel de la investigación como motor de cambio, innovación e indicador de calidad de un sistema educativo, que debe impregnar a todos los niveles del mismo. Algunos países vienen desarrollando investigaciones desde tiempos lejanos -Colombia (1930), México (1954), Venezuela y Uruguay (1959), Chile (1960)... -, pero la realidad es que, como ya apuntábamos en el apartado anterior, al describir las colaboraciones internacionales, son pocos los países que implementan una investigación sistemática en los diversos niveles del sistema educativo y con objetivos que abarquen al mismo en su globalidad. Los objetivos se reflejan sobre el papel, pero en la práctica son limitadas.

Un número limitado de países manifiestan una estrecha colaboración con las universidades -México, Brasil, Argentina, Uruguay-; los más, la colaboración es escasa -Chile-, cuando no nula.

En algunos países, la investigación se genera desde las aulas -Panamá-, pero lo habitual es que sea centralizada -Venezuela, a través del CONICIT, Costa Rica, Cuba,...- , en algún caso desde los organismos intermedios -Argentina, en las unidades provinciales, o dando su apoyo a los Consejos Desconcentrados -Uruguay-.

La conclusión en este capítulo es que la labor a realizar es ingente: hay que pedir colaboración a los organismos internacionales, dar soporte técnico, económico y, primeramente, político, a los niveles inferiores, orientar las investigaciones al aula, en el nivel inferior, investigaciones de planeamiento -prioritariamente las que se refieren a las transferencias tecnológicas, en los niveles intermedios y, por último, la indagación sistemática y autocrítica desde el nivel superior.

d) Por último, por la brevedad del espacio que así nos lo demanda, sería el paso de la descentralización administrativa a la autonomía de los centros. Nuevamente, las diversas crisis económicas y políticas-, llevan a la centralización del sistema educativo -países centroamericanos, pero también Chile, México, etc-. Pero lo habitual en la mayoría de los países es que convivan niveles y ámbitos centralizados con otros descentralizados -Cuba "descentralización planificada", Venezuela, Perú "descentralización de la gestión administrativa", Uruguay apuesta por la planificación estratégica "una realidad no del todo acabada", Bolivia "planificación normativa en el ámbito estatal y descentralización técnico-pedagógica", Argentina, descentralización en las funciones, pero no en la autonomía.

Pero algunos países están en la línea, cuando menos teórica de apostar por la autonomía escolar: Chile "descentralización pedagógica", Nicaragua "fortalecimiento de la autonomía escolar", Ecuador "Sistema Integral de Evaluación Educativa", Panamá, el SINICE quiere "fortalecer los centros escolares" y Brasil que "organiza la evaluación como un sistema integrado que abarca diferentes niveles de enseñanza [...] que auxilia en el dia a dia escolar".

Todos los países, como hemos visto, en un grado $u$ otro, se debaten entre la centralización y la descentralización. ¿Qué conviene en el marco socioeconómico actual?. Apostar por la descentralización entendida como una variedad de formas organizativas, con objetivos diversos, con autoridad propia y que tiene como finalidad última la autonomía de cada uno de los 
niveles. Las relaciones que deben darse en este marco descentralizado son las de intercambio, de interdisciplinariedad. La descentralización real y eficaz debe ser fruto de una cultura de participación y responsabilidad civil -las CEFFAs son un ejemplo a seguir y apoyar-, que necesita un proceso de revitalización y compromiso constante, en el que la escuela sea el centro del sistema educativo. Esta autonomía de los centros ha de fundamentarse en la estrategia del compromiso, a través de formas colegiadas y comunitarias de organización escolar.

En la historia se hace lo que se puede y no lo que se quiere hacer. Una de las grandes tareas políticas que hemos de llevar a cabo es la persecución constante de hacer posible mañana aquello que hoy es imposible (Paulo Freire, 1992).

\section{BIBLIOGRAFÍA}

Driessen, G. "La mejora de la igualdad y la calidad en la enseñanza: el caso de Holanda". Revista de Educación, nº.319. Madrid: MEC. (1999)

Ferranti y Perry: "La brecha educativa en América Latina". La Vanguardia, 14 de noviembre del 2002

Gipps, C. "La evaluación del alumno y el aprendizaje en una sociedad en evolución". Perspectivas, vol.XXVIII, $n^{\circ}$.1. París: UNESCO. (1998)

Herpen, M. van "Les indicateurs de l'enseignement: modèles théoriques". L'OCDE et les indicateurs internationaux de l'enseignement: una cadre d'analyse. Paris: OCDE. (1992)

Kallen, D. "Nouveaux paradigmes éducatifs et nouvelles politiques d'évaluation". Évaluer et réformer les systèmes éducatifs. Paris: OCDE. (1996)

Mateo, J. "L’avaluació de les institucions educatives". I Jornadas sobre Medición y Evaluación Educativas: Estándares e Indicadores para analizar la realidad educativa. Valencia, marzo. (2000)

OCDE. Education at a Glance. Paris: OCDE. (1994,1998)

OCDE Gros pla sur les écoles. Paris: OCDE. (1995)

Schriewer, J. "Sistema mundial y redes de interrelación: la internacionalización de la educación y el papel de la investigación comparada". Globalización y descentralización de los sistemas educativos. Barcelona: Pomares-Corredor. (1996)

Tiana, A. Avaliaçâo Educacional: Cooperación internacional en evaluación de la educación en América Latina y el Caribe: análisis de la situación y propuestas de actuación. Cúpula das Américas - Linha 2. INEP. Ministerio Da Educaçâo. (2000)

Wolff, L.: Las Evaluaciones Educacionales en América Latina: Avance Actual y Futuros Desafíos. Whashington \& Santiago de Chile: PREAL (1998) 


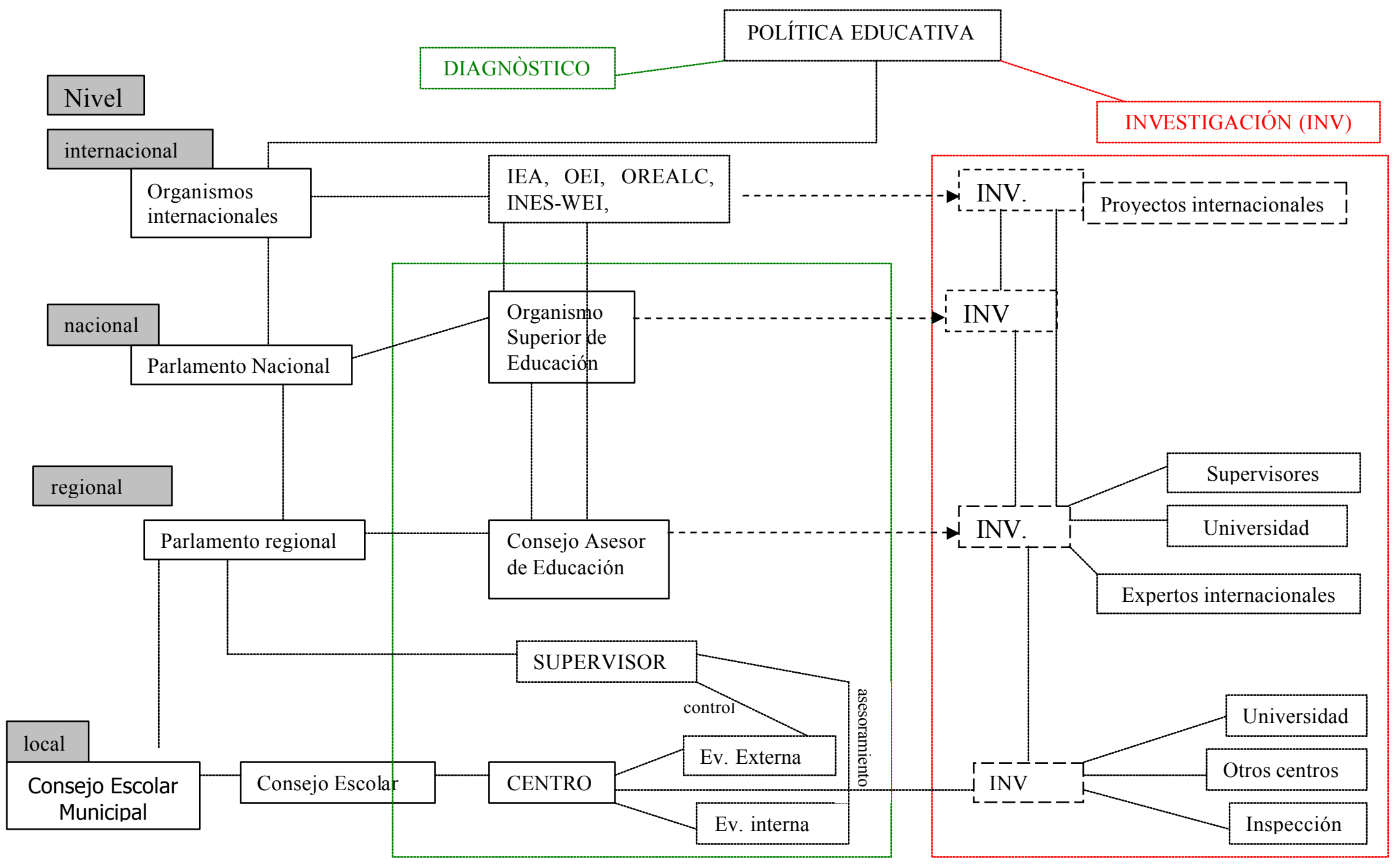

